\title{
PARTICIPACIÓN Y APOYO DE LA FAMILIA INDÍGENA EN LOS PROCESOS EDUCATIVOS
}

\author{
Carlos Miranda Carvajal ${ }^{1}$ \\ Pablo Castillo Armijo ${ }^{2}$
}

\begin{abstract}
RESUMEN: El diseño del estudio corresponde al tipo cuantitativo no experimental, de carácter descriptivo. Como variables se evaluaron dimensiones de comunicación, apoyo y conocimiento de la escuela. Se buscaron correlaciones con la etnia indígena, vulnerabilidad escolar y adulto responsable como jefe de hogar. Se puede afirmar que el apoyo y participación de las familias pertenecientes a las etnias indígenas es más alto que las familias que no pertenecen a ninguna etnia. También se puede establecer que las familias que muestran una mayor participación y apoyo familiar en los procesos educativos son las que se constituyen con un adulto responsable del estudiante.
\end{abstract}

Palabras-clave: Relación padres-escuela. Vulnerabilidad escolar. Grupo étnico.

\section{PARTICIPATION AND SUPPORT OF THE INDIGENOUS FAMILY IN EDUCATIONAL PROCESSES}

\begin{abstract}
This study is non-experimental quantitative, of a descriptive nature. We evaluated variables, dimensions of communication, support, and knowledge of the school. Correlations between the indigenous ethnic group, the school vulnerability, and a head of household adult were researched. It is possible to state that the support and the participation of the families belonging to the indigenous ethnic groups are higher than those of the nonethnic families. It can also be established that the families that show greater participation and family support in the educational processes are those which have an adult responsible for the student.
\end{abstract}

Keywords: Parent-school relation. School vulnerability. Ethnic group.

1. Pontifica Universidad Católica de Valparaíso - Escuela de Psicología - Programa de Doctorado en Psicología - Valparaíso, Chile. E-mail: carlos.miranda.c01@mail.pucv.cl

2.Universidad Católica del Maule - Departamento de Fundamentos de la Educación - Facultad de Ciencias de la Educación - Talca, Chile.E-mail: pcastillo@ucm.cl 


\title{
PARTICIPAÇÃO E APOIO À FAMÍLIA INDÍGENA NOS PROCESSOS EDUCATIVOS
}

\begin{abstract}
RESUMO: Este é um estudo quantitativo não experimental, de caráter descritivo. Como variáveis, foram avaliadas dimensões de comunicação, apoio e conhecimento da escola. Buscaram-se correlações entre a etnia indígena, a vulnerabilidade escolar e o adulto considerado chefe da família. Pode-se afirmar que o apoio e a participação das famílias pertencentes às etnias indígenas são mais altos que os das famílias não étnicas. Também podemos estabelecer que as famílias que mostram maiores participação e apoio familiares nos processos educativos são as que mais possuem um adulto como responsável pelo estudante.
\end{abstract}

Palavras-chave: Relação pais-escola. Vulnerabilidade escolar. Grupo étnico.

\section{Introducción}

$\mathrm{E}$

xisten diversos factores reconocidos como efectivos para el cambio y mejora escolar. En este artículo, nos centraremos en el apoyo familiar y más específicamente en familias que se son reconocidas como indígenas en el contexto chileno.

Destacamos las recomendaciones entregadas por Castillo et al. (2017), Elboj (2001), Elboj et al. (2002), Molina (2007), Valls (2000) y Vieira (2011) en relación a la construcción de una escuela como una comunidad de aprendizaje (CdA), donde el factor de apoyo familiar es fundamental para lograr mejores resultados de aprendizajes.

En el caso chileno la Agencia de la Calidad de la Educación (2015) de Chile plantea que la familia es un pilar importante del logro de la anhelada calidad educativa, y es por esta razón que se toma como antecedente para analizar los resultados del Sistema de Medición de la Calidad de la Educación (SIMCE).

La importancia del crecimiento sano y bienestar de los niños y niñas hace imperiosa que la relación comunidad-familia se involucren en los procesos educativos y que las escuelas se abran a la comunidad local en la que ésta misma se encuentra (CASTILLO et al., 2017; UNESCO, 2012).

Si se considera a la familia como un grupo social, unidos entre sí por vínculos de consanguinidad, filiación (biológica o adoptiva) y de alianza, incluyendo las uniones de hecho cuando son estables (GARCIA, 2009, p. 16) y bajo ella las propuestas de participación en la educación en forma de crianza, comunicación con la escuela, voluntariado, toma de decisiones, colaboración con la comunidad y supervisión del aprendizaje en el hogar (EPSTEIN; SHELDON, 2008), llevadas a las familias que poseen la calidad de indígenas de los distintos pueblos del Chile precolombino y la realidad educativa municipal, es que podemos plantear como objetivo del trabajo Conocer cuantitativamente el nivel de Apoyo y Participación Familiar en los procesos educativos de los estudiantes, según las características propias de la Familia, jefe de hogar y pertenencia indígena e índice de vulnerabilidad escolar.

\section{Marco Teórico}

Sobre la composición familiar indígena, es necesario entender la etnicidad como una relación social que ocurre en un contexto particular, transitorio y temporal, a nivel nacional y que, también se erige socialmente en espacios de fronteras culturales (ARAVENA, 2003), a micro escala, siendo las familias las 
que actúan como núcleos particulares de conservación y pertenencia de sus componentes y características comunes.

La composición familiar de los pueblos indígenas, se rige, al igual que la visión social que poseen por el concepto de cosmovisión, algo propio de cada pueblo, pero que a la vez es común y particular, ya que, cada pueblo posee una propia cosmovisión que lo identifica y diferencia del resto del mundo, es la forma de interpreta su propia existencia en una sociedad y que a la vez los une con el origen y la figura divina representada en la madre (MAMANI; ARAYA, 2015).

Las formas en que se asume en un centro urbano el sentido de pertenencia a alguno de los pueblos indígenas, se puede dimensionar a nivel de comunidad, como una expresión parental y de participación dentro de la organización. La acción colectiva de la familia representa por medio de la cooperación y la preservación de sus cosmovisiones, no solo involucra a una familia nuclear, más bien está compuesta por una amplia línea genealógica desde los ancestros (ARAVENA, 2003), denominados en las culturas andinas como "los abuelos" (ARAYA; SEGOVIA, 2010; MAMANI; ARAYA, 2015).

Los denominados vínculos primordiales por Morales (2016), revisten ciertas características típicas: se presentan como "datos" a priori rebeldes a todo análisis; son percibidos como algo inefable, poderoso, coercitivo y, sobre todo, son capaces de generar fuertes emociones y afectos, los que también son planteados como "saber informal" o "patrimonio local" (MIRANDA, 2017).

La mirada actual sobre esta descripción de saberes, es la que se observa en la investigación como primera fuente de educación a nivel de familias indígenas, donde Araya y Segovia (2010) usan esta fuente de conocimiento para representar los orígenes de las historias ancestrales culturales de los primeros asentamientos indígenas en las que hoy son grandes ciudades, y que Montecino (1996) usa para representar la fundación del mestizaje como producto y productor de una cultura indígena nueva con representaciones europeas que toman significados desde las cosmovisiones indígenas. Sin embargo, las relaciones de apoyo indígena familiar a la labor educativa de escuelas convencionales han sido poco estudiada a pesar del impacto de traspaso de cultura y cosmovisión que de ellas se reconoce.

La investigación sobre la efectividad escolar ha entregado diversas conclusiones que apoyan los procesos de enseñanza-aprendizaje y permiten el logro de altos resultados académicos. Estos factores que componen el modelo de efectividad escolar se presentan como factores asociados al logro de aprendizajes escolares y que pueden impactar en el buen funcionamiento de la escuela, independiente de su nivel socioeconómico (ARANCIBIA, 1992; BELLEI et al., 2014; 2015; CORNEJO; REDONDO, 2007; HIMMEL et al., 1984; LLECE; UNESCO, 2002; MURILLO, 2007; PIZARRO; CLARK, 1987, 1998; UNESCO, 2008; UNICEF, 2004; ZÁRATE, 1992).

La investigación contemporánea en Chile ha puesto los ojos en el funcionamiento interno y externo que poseen las instituciones educativas en la búsqueda de buenos resultados académicos. Entre los aspectos valorados se encuentran elementos culturales, morales y éticos de los docentes en conjunto con el compromiso institucional, ya no solo con los estudiantes, sino también en su relación con sus familias, y teniendo efectos en la mejora de los aprendizajes (BELLEI et al., 2015).

Tanto la familia como la escuela son instituciones pilares del desarrollo y convivencia humana (BELMAR, 2003; CANO; CASADO, 2015), por lo que el apoyo familiar ha sido un elemento primordial en los procesos educativos y éste se refleja directamente en el grado de involucramiento de los padres o adultos responsables de los estudiantes en las distintas actividades académicas, produciendo un efecto positivo en el desempeño cuantitativo de los estudiantes (BAZÁN et al., 2007).

La composición del apoyo familiar en las labores educativas, se expresa claramente en las definidas como variables de la escuela y variables origen de los estudiantes. Éstas últimas se clasifican en variables 
de la comunidad de origen, variables del hogar de origen (BRUNNER; ELACQUA, 2004 apud CORNEJO; REDONDO, 2007; GERSTENFELD, 1995) y componentes del currículo del hogar (PIZARRO, 2006, 2009; PIZARRO; CLARK, 1987, 1998). Propuesto desde la visión de estas dos grandes variables poseen influencias en los aprendizajes de los estudiantes, las que pueden ser positivas o negativas. Según estos mismos autores la escuela tiene mayormente influencias positivas, sin embargo, la variable origen y su composición posee un igual porcentaje influencias positivas y negativas en los logros escolares.

En las investigaciones sobre cómo realmente se incluye a la familia en los procesos de enseñanza y aprendizaje, también se pueden encontrar opiniones que difieren de su importancia. Una visión más positiva de esta participación, puede mostrar la inclusión de la familia en los procesos educativos como un constructo particular, que puede no sólo observase como un factor asociado de algún modelo educativo como la escuela efectiva (ARANCIBIA, 1992; BELLEI et al., 2015, 2014; HIMMEL et al., 1984; LLECE; UNESCO, 2002; MURILLO, 2007; UNESCO, 2008; UNICEF, 2004; ZÁRATE, 1992).

Una indiscutible participación familiar en la escuela se transforma en el núcleo de la mejora de los procesos educativos (TEDESCO, 2000 apud ANABALÓN et al., 2008), expresada según diversas variables que se desarrollan teóricamente por variados autores (EPSTEIN; SHELDON, 2008; PIZARRO, 2006, 2009; RUIZ, 2003; VALDÉS et al., 2009, 2013; YURÉN; CRUZ, 2009; VIEIRA, 2011), y planteadas de la siguiente manera: crianza, colaboración con la comunidad, toma de decisiones, voluntariado, comunicación con la escuela, fomento al cumplimiento de metas, influencia en la deserción escolar, y la ayuda y apoyo en las tareas.

La investigación cuantitativa desarrollada en relación a la familia y su influencia en los procesos educativos muestra una relación directa entre los logros académicos y el denominado currículum del hogar (PIZARRO, 2006, 2008, 2009, 2015), donde la variable de apoyo al aprendizaje en el hogar se expresa como Guía y Apoyo Académico.

De esta forma la guía y apoyo académico en los Índices de Riesgos Educativos (IREs) se observa para un $\mathrm{N}=1.636$ pesos factoriales que se acompañan del Currículum del Hogar Mejorado:

Los logros de Lenguaje están determinados en un $2^{\circ}$ lugar por la autoestima y currículum del hogar, los siguientes resultados son mezclas de factores que favorecen el logro académico creciente. El mismo fenómeno se encuentra en los logros de asignaturas como Matemáticas, donde una vez más, la autoestima se acompaña del currículum del hogar en el 2o lugar de los pesos factoriales (FOLIACO et al., 2006; PIZARRO, 2009).

Los resultados obtenidos en el análisis de los IREs se muestran estadísticamente obtenidos por la aplicación del cuestionario de Pizarro 2005-R, donde para N = 1372 la dificultad fue 68,64, por lo que se asume que las familias que componen la muestra cumplen un rol positivo y apegado a las correctas interacciones educativas, psicológicas y sociológicas (PIZARRO, 2009).

La importancia del crecimiento otorgado desde la estrategia Educativa de Currículum del Hogar, expresada en el aumento de tamaño expresado en Delta de Glass +0,75 sigmas (PIZARRO et al., 1997, 1998; PIZARRO, 2008), muestra la importancia de las propuestas teóricas en relación a los avances en los resultados académicos.

\section{Metodología}

Se consideró como variable dependiente al Apoyo y Participación Familiar en los Procesos Educativos según la propuesta de Miranda y Castillo (2018), que plantean la representación de 3 variables: Comunicación con la Escuela, Apoyo y Supervisión del Aprendizaje en el Hogar y Conocimiento de la Escuela. 
Para la realización de las correlaciones según las características de la familia de los estudiantes se tomó en cuenta la etnia indígena, considerando las diferentes etnias ${ }^{1}$ que existen en el territorio chileno. De la misma manera, se determina una aproximación al nivel socioeconómico de las familias y de la realidad escolar en que se considera el adulto responsable como jefe de hogar y el índice de vulnerabilidad escolar (IVE) de los establecimientos seleccionados en la muestra y entregado por el Sistema Nacional de Asignación con Equidad (SINAE) ${ }^{2}$ que maneja la Junta Nacional de Auxilio Escolar y Becas (JUNAEB) en Chile.

El diseño del estudio corresponde al tipo cuantitativo no experimental, de carácter descriptivo, desarrollando un análisis multivariado de las variables (se consideraron tres factores en el instrumento aplicado), presentes dentro de un constructo específico (KERLINGER; LEE, 2002; MARRADI et al., 2012; PIZARRO, 2012). Para el tratamiento de la información, se aplicaron pruebas estadísticas usando el software SPSS Statistics versión 21 para IOS.

La muestra con la que se desarrolló el estudio fue de $\mathrm{N}=520$ apoderados de $6^{\circ}$ año básico, esta muestra equivale a un $38,14 \%$ de la población del citado curso en la totalidad de los establecimientos educacionales de carácter municipal de la ciudad de Calama.

Para la recolección de datos sobre las variables seleccionadas se utilizó un instrumento tipo Likert, tomando como base la propuesta de Valdés, Carlos y Arreola (2013). Se redactaron 32 ítems de los cuales se establecieron tres categorías que corresponden a 12 preguntas para Comunicación con la Escuela, 12 preguntas para Apoyo y Supervisión del Aprendizaje en el Hogar y 8 preguntas para Conocimiento de la Escuela (Tabla 1).

Tabla 1. Especificaciones del cuestionario.

\begin{tabular}{ccc} 
Categorías & Ítems & No de la Pregunta \\
\hline Comunicación con la Escuela & 12 & 1 a 12 \\
\hline Apoyo y Supervisión del Aprendizaje en el Hogar & 12 & 13 a 24 \\
\hline Conocimiento de la Escuela & 8 & 25 a 32 \\
\hline
\end{tabular}

Fuente: Elaboración propia.

La validación del cuestionario (Tabla 2) se realizó por medio de una aplicación experimental de testing en niveles de $1^{\text {os }}$ y $2^{\text {os }}$ básicos de tres establecimientos educativos municipales y particulares subvencionados, pertenecientes a las comunas de Viña del Mar, Valparaíso y Santiago. La muestra inicial fue de 318 estudiantes, de los cuales se contestaron 247 cuestionarios, que representa el 77,67\% de la población.

Tabla 2. Estadísticos de la confiabilidad.

\begin{tabular}{ccc}
\hline Alfa de Cronbach & Alfa de Cronbach Basada en los Elementos Tipificados & No de Elementos \\
\hline 0,810 & 0,840 & 32 \\
\hline
\end{tabular}

Fuente: Elaboración propia.

Al determinar la confiabilidad del cuestionario para Padres y Apoderados aplicada según Alfa de Cronbach y la categorización la confiabilidad, obtenemos el nivel de interpretación EXCELENTE, considerando que el grado de significancia usado fue de 99,9 (CARO et al., 2002; KERLINGER; LEE, 2002; NUNNALLY, 1970, 1987; PIZARRO, 2012). Existe un 81\% de similitud entre los tres desempeños de las subvariables sintetizadas y el puntaje total de Participación y Apoyo en los Procesos Educativos.

La confiabilidad total del instrumento se calculó según Alfa de Cronbach considerando $\mathrm{N}=520$, obteniendo como resultado una mejora en relación a la aplicación experimental del mismo (Tabla 3). El 
valor Alfa de Cronbach, obtenido por medio de la aplicación del mismo cuestionario, en dos etapas, atribuye al aumento del $\mathrm{N}$ que rindió el cuestionario un grado significante en el cálculo en relación a la aplicación experimental con $\mathrm{N}=247(\alpha=0,810)$, se observa un aumento de 0,091 en la aplicación real teniendo $\mathrm{N}=520$ $(\alpha=0,901)$. Ello permite clasificar como EXCELENTE a este grado de confiabilidad, pudiendo medir de mejor manera el constructo y las variables seleccionadas, además del "computo de Análisis Factorial comprobatorio $(\mathrm{AFc})$, donde se obtuvieron resultados para la adecuación muestral $\mathrm{KMO}=0,870$ y un $62,0 \%$ residuales redundantes con valores absolutos menores que 0,05 , observándose una real asociación entre las teorías supuestas y las respuestas obtenidas en el cuestionario (validez de constructo)" (MIRANDA; CASTILLO, 2018, p. 130)

Tabla 3. Confiabilidad del cuestionario.

\begin{tabular}{ccc}
\hline Alfa de Cronbach & Alfa de Cronbach Basada en los Elementos Tipificados & No de Elementos \\
\hline 0,901 & 0,906 & 32 \\
\hline
\end{tabular}

Fuente: Elaboración propia.

\section{Análisis y Resultados}

\section{Participación Familiar en los Procesos Educativos según Etnia Indígena}

Del total de la muestra representado con un $\mathrm{N}=520$, un 22,11\% declara pertenecer a etnias indígenas, que se ordenan de mayor a menor según el número de encuestas contestadas como sigue Atacameña, Aymara, Quechua, Diaguita, Mapuche. Esta presencia intercultural no es común de encontrar en otras regiones de Chile, y por tanto representa una singularidad del contexto estudiado.

Para la categoría denominada Apoyo y Supervisión del Aprendizaje en el Hogar se obtuvo un puntaje para las Familias participantes ordenados de mayor a menor es: Atacameño $(112,50)$, Quechua $(111,65)$, Aymara $(108,26)$, Mapuche $(107,71)$ y Diaguita $(101,63)$; en donde la media para Etnia Indígena es 108,35 y para Sin etnia es de 109,80. (Tabla 4)

Tabla 4. Puntaje TOTAL según etnia indígena.

\begin{tabular}{cccc}
\hline Etnia Indígena & Media Aritmética & No $^{\mathbf{2}}$ & Desviación Típica \\
\hline Atacameña & 112,50 & 64 & 9,822 \\
\hline Quechua & 111,65 & 17 & 10,571 \\
\hline Aymara & 108,26 & 19 & 13,856 \\
\hline Mapuche & 107,71 & 7 & 11,884 \\
\hline Diaguita & 101,63 & 8 & 10,309 \\
\hline Subtotal etnia & 108,35 & 115 & 13,404 \\
\hline Sin etnia & 109,80 & 405 & 12,918 \\
\hline Total & 109,98 & 520 & \\
\hline
\end{tabular}

Fuente: Elaboración propia.

A modo de resumen los puntajes alcanzados por etnia y su relación al $\mathrm{N}$ se expresa de la siguiente manera: Atacameño = 112,50 sobre un $\mathrm{N}=64$; Quechua $=111,65$ sobre un $\mathrm{N}=17$; Aymara = 108,26 sobre un $\mathrm{N}=19 ;$ Mapuche $=107,71$ sobre un $\mathrm{N}=7$; Diaguita $=101,63$ sobre un $\mathrm{N}=8$. 


\section{Participación Familiar en los Procesos Educativos según Índice de Vulnerabilidad Escolar}

El índice de vulnerabilidad y apoyo y supervisión del aprendizaje en el hogar se expresan según la clasificación dada por el MINEDUC y usada por la JUNAEB (Junta Nacional de Auxilio Escolar y Becas) en la clasificación de los establecimientos educacionales, sobre el $\mathrm{N}=520$ no se encuentran valores perdidos, ya que la asignación de puntaje se realizó a cada uno de los casos según el establecimiento al cual pertenece el apoderado a quién se aplicó el instrumento. Es necesario considerar que se incluyeron cinco valores, y uno de ellos agrupa a dos establecimientos con el índice de menor valor (Tabla 5).

Tabla 5. Informe de TOTAL según Índice de Vulnerabilidad.

\begin{tabular}{cccc}
\hline Índice Vulnerabilidad & Media Aritmética & No & Desviación Típica \\
\hline 40,0 & 108,67 & 146 & 11,971 \\
\hline 53,8 & 113,15 & 55 & 10,368 \\
\hline 57,6 & 108,08 & 66 & 13,876 \\
\hline 65,9 & 109,53 & 171 & 14,285 \\
\hline 73,2 & 112,70 & 82 & 11,727 \\
\hline Total & 109,98 & 520 & 12,918 \\
\hline
\end{tabular}

Fuente: Elaboración propia.

El índice de vulnerabilidad escolar ordenado de mayor a menor es:

1. Índice de vulnerabilidad $=73,2 \%$ con $\mathrm{N}=82$;

2. Índice de vulnerabilidad $=65,9 \%$ con $\mathrm{N}=171$;

3. Índice de vulnerabilidad $=57,6 \%$ con $\mathrm{N}=66$;

4. Índice de vulnerabilidad $=53,8 \%$ con $\mathrm{N}=55$;

5. Índice de vulnerabilidad $=40,0$ con $\mathrm{N}=146$.

La asignación considera un $100 \%$ de los casos estudiados con total de la muestra $\mathrm{N}=520$. Según el puntaje alcanzado para Total el orden de mayor a menor es:

1. Índice de vulnerabilidad $=53,8 \%$ con media aritmética Total $=113,15$;

2. Índice de vulnerabilidad $=73,2 \%$ con media aritmética Total $=112,70$;

3. Índice de vulnerabilidad $=65,9 \%$ con media aritmética Total $=109,53$;

4. Índice de vulnerabilidad $=40,0 \%$ con media aritmética Total $=108,67$;

5. Índice de vulnerabilidad $=57,6 \%$ con media aritmética Total $=108,08$.

\section{Participación Familiar en los Procesos Educativos según Características de la Familia}

El apoyo y supervisión del aprendizaje en el hogar detallado por la composición familiar, entendida como el adulto responsable del estudiante, donde no se observaron valores perdidos, por lo que el $\mathrm{N}=520$ equivalentes al 100\% de casos revisados obteniendo los siguientes resultados (Tabla 6).

Un orden de mayor a menor según $\mathrm{N}$ por características de la Familia considerando al adulto responsable del estudiante fue: Padres con $\mathrm{N}=278$, Madre con $\mathrm{N}=186$, Padre con $\mathrm{N}=27$, Abuelos con $\mathrm{N}=14$, Hermana con $\mathrm{N}=6$, Tíos con $\mathrm{N}=5$, Abuela con $\mathrm{N}=3$, Abuelo con $\mathrm{N}=1$, donde los $\mathrm{N}$ correspondientes a los dos primeros grupos corresponden al 53,461\% y 35,769\% respectivamente, cubriendo entre ambos un $89,23 \%$ de la muestra total. 
Tabla 6. Informe de TOTAL según Adulto Responsable del Estudiante.

\begin{tabular}{cccc} 
Adulto Responsable & Media Aritmética & No & Desviación Típica \\
\hline Abuela & 117,67 & 3 & 9,074 \\
\hline Abuelo & 111,00 & 1 & 0,00 \\
\hline Abuelos & 117,79 & 14 & 5,912 \\
\hline Hermana & 76,83 & 6 & 8,256 \\
\hline Madre & 108,74 & 186 & 13,983 \\
\hline Padres & 111,72 & 278 & 11,198 \\
\hline Padre & 101,00 & 27 & 10,359 \\
\hline Tíos & 121,20 & 5 & 9,960 \\
\hline Total & 109,98 & 520 & 12,918 \\
\hline
\end{tabular}

Fuente: Elaboración propia.

El puntaje para Total apoyo y supervisión del aprendizaje en el hogar ordenado de mayor a menor es: Tíos $=121,20 ;$ Abuelos =117,79; Abuela = 117,67; Padres = 111,72; Abuelo = 111,00; Madre = 108,74; Padre $=101,00$; Hermana $=76,83$.

$\mathrm{Al}$ revisar las características de las familias con mayor presencia y participación en los procesos educativos, podemos sostener que los $\mathrm{N}$ de los tipos de familia que poseen los tres mayores puntajes son mínimos en relación al $\mathrm{N}$ total de la muestra. Por su parte los tipos de familia con mayor $\mathrm{N}$ se encontraron en un nivel medio de participación entre las 8 formas de familias caracterizados según la realidad estudiada.

\section{Consideraciones Finales}

Para Conocer el nivel de Apoyo y Participación Familiar según las características propias de la familia, jefe de hogar y pertenencia indígena, se observó a la familia y sus características particulares para identificar dos de ellas que pueden hacer mayores diferencias, la primera es la pertenencia a etnia indígena, y la segunda quién es el jefe del hogar donde vive el estudiante.

Referente a la pertenencia a alguna etnia indígena, el 22,11\% de la muestra declara pertenecer a alguno de los pueblos indígenas obteniendo una media aritmética en puntaje que representa un $84,64 \%$ de logro en referencia al puntaje ideal. Por ello, se considera que las familias pertenecientes a una etnia indígena poseen un alto índice de Apoyo y Participación Familiar en los Proceso Educativos. En los distintos pueblos, las familias pertenecientes a la etnia Atacameña con logro de 87,89\%, son quiénes muestran mayor Apoyo y Participación Familiar, seguido por las familias del pueblo Quechua con $87,23 \%$, las familias del pueblo Aymara con $84,58 \%$, las familias del pueblo Mapuche con $84,15 \%$ y las familias del pueblo Diaguita con 79,40\%.

Si bien se observan diferencias entre los distintos pueblos a los que pertenecen las familias y estudiantes de la muestra, se puede afirmar que el Apoyo y Participación de las Familias pertenecientes a las etnias indígenas, es significativamente más alto que el Apoyo y Participación de las familias que no pertenecen a ninguna etnia.

También se encontró con que las familias que muestran una mayor Participación y Apoyo Familiar en los Procesos Educativos son las que poseen como adulto responsable del estudiante a: tíos, abuelos, abuela, padres, abuelo, madre, padre y hermana. La concentración de mayor $\mathrm{N}$ está en los grupos de adulto responsable, padres y madre con un $\mathrm{N}=464$ correspondiente al $89,23 \%$ de la muestra total. Las familias que 
poseen como jefe de hogar a la madre con puntaje $=108,74$ igual a un $84,95 \%$ del puntaje ideal; padre con puntaje $=101,00$ igual a un $78,90 \%$ del puntaje ideal, padres con puntaje $=111,72$ igual a un $87,27 \%$ del puntaje ideal. Podemos sostener que la Participación y Apoyo Familiar en los Procesos Educativos es ALTA considerando que los logros porcentuales superan el 75\% en los tipos de familia en la realidad estudiada.

Para establecer los niveles de Apoyo y Participación Familiar según los índices de vulnerabilidad de las escuelas municipales de la ciudad de Calama, los puntajes alcanzados para Participación Familiar corresponden en un orden determinado, según mayor o menor índice de vulnerabilidad escolar, ya que los mayores puntajes para esta caracterización hacen mención a las familias más pobres a nivel económico y social, donde el mayor índice de vulnerabilidad $(73,2)$ alcanza un puntaje $88,04 \%$. Por su parte el menor índice de vulnerabilidad obtenido $(40,0)$ corresponde al $84,89 \%$ del puntaje. Se destaca que los extremos en los índices de vulnerabilidad poseen una mejor Participación y Apoyo Familiar en los Procesos Educativos. Es importante considerar que los niveles medios de vulnerabilidad escolar (entre 50 y 60 ) no se consideran bajos, pero sus valores están bajo los valores extremos para este indicador.

\section{Contribución de los Autores}

Problematización y Conceitualización: Castillo P, Miranda C; Metodología: Miranda C; Castillo P; Análisis: Miranda C; Castillo P; Redacción: Castillo P; Miranda C.

\section{Notas}

1. La Corporación Nacional de Desarrollo Indígena (CONADI) reconoce las siguientes etnias a las cuales se puede pertenecer en Chile: Aymara, Rapa Nui (o pascuense), Atacameña, Quechua, Colla, Diaguita, Alacalufe (o kawashkar), Yagán (o yámana) y Mapuche.

2. El Sistema Nacional de Asignación con Equidad (SINAE) es un conjunto de criterios que permite identificar distintos grupos dentro de la población de estudiantes de educación básica y media de establecimientos municipales o particulares subvencionados del país, de acuerdo al nivel de vulnerabilidad que presentan. Así, la población identificada como "vulnerable" queda clasificada en 3 prioridades, siendo la primera prioridad el grupo que reúne a los estudiantes con riesgos principalmente socioeconómicos; la segunda prioridad un grupo con menor vulnerabilidad socioeconómica, pero que además presenta riesgos socioeducativos asociados a problemas de rendimiento escolar, asistencia o deserción del sistema educacional; y, finalmente, la tercera prioridad reúne a estudiantes con el mismo nivel de vulnerabilidad socioeconómica que la segunda prioridad pero que no presenta problemas como los identificados en la segunda prioridad.

\section{Referencias}

AGENCIA DE LA CALIDAD DE LA EDUCACIÓN. La calidad educativa desde la percepción de los actores claves del sistema, 2015. Disponible en: http://www.agenciaeducacion.cl/wp-content/uploads/2016/02/ Estudio_Calidad_educativa_percepcion_actores_clave_del_sistema.pdf. Acceso en: 22 mar. 2018.

ANABALÓN, M. et al. El compromiso familiar frente al desempeño escolar de niños y niñas de educación general básica en la ciudad de Chillán. Revista Horizontes Educacionales, v. 13, n. 1, p. 11-21, 2008. Disponible en: http://www.redalyc.org/articulo.oa?id=97912446001. Acceso en: 25 mar. 2018. 
ARANCIBIA, V. Efectividad escolar: Un análisis comparado. Estudios Públicos, n. 47, p. 101-125, 1992. Disponible en: https://www.cepchile.cl/efectividad-escolar-un-analisis-comparado/cep/2016-03-03/184840. html. Acceso en:02 abr. 2018.

ARAVENA, A. El rol de la memoria colectiva y de la memoria individual en la conversión identitaria mapuche. Estudios Atacameños, n. 26, p. 89-96, 2003.

ARAYA, E.; SEGOVIA, W. Historia ancestral oral y cultural de la población la isla de La Banda, Calama. Antofagasta: CORE, 2010.

BAZÁN, A.; SÁNCHEZ, B.; CASTAÑEDA, S. Relación estructural entre el apoyo familiar, nivel educativo de los padres, características del maestro y desempeño en la lengua escrita. Revista Mexicana de Investigación Educativa, v. 12, n. 33, p. 701-729, 2007. Disponible en: http://www.redalyc.org/articulo.oa?id=14003312. Acceso en: 02 abr. 2018.

BELLEI, C. et al. Lo aprendí en la escuela: ¿Cómo se logran procesos de mejoramiento escolar? Santiago: Universidad de Chile, 2014.

BELLEI, C. et al. Nadie dijo que era fácil. Escuelas efectivas en sectores de pobreza, diez años después. Santiago: LOM Ediciones, 2015.

BELMAR, B. Familia y escuela: Realidades y desafíos. Revista Horizontes Educacionales, n. 8, p. 7-14, 2003. Disponible en: http://www.redalyc.org/articulo.oa?id=97917166002. Acceso en: 05 abr. 2018.

CANO, R.; CASADO, M. Escuela y familia: Dos pilares fundamentales para unas buenas prácticas de orientación educativa a través de las escuelas para padres. Revista Electrónica Interuniversitaria de Formación del Profesorado, v. 18, n. 2, p. 15-28, 2015. https://doi.org/10.6018/reifop.18.2.219491

CARO, A.; ESCALANTE, E.; BARAHONA, L. Análisis y tratamiento de datos en SPSS. Valparaíso: Ediciones Universidad de Playa Ancha, 2002.

CASTILLO, P.; PUIGDELLIVOL, I.; ANTÚNEZ, S. El liderazgo compartido como factor de sostenibilidad del proyecto de comunidades de aprendizajes. Revista Estudios Pedagógicos, v. 43, n. 1, p. 41-59, 2017. https://doi.org/10.4067/S0718-07052017000100003

CORNEJO, R.; REDONDO, J. Variables y factores asociados el aprendizaje escolar: Una discusión desde la investigación actual. Revista Estudios Pedagógicos, v. 33, n. 2, p. 155-175, 2007. https://doi.org/10.4067/ S0718-07052007000200009

ELBOJ, C. Comunidades de aprendizaje: Un modelo de educación antiracista en la sociedad de la información. 2001. 339 f. Tesis (Doctorado en Estructura y Cambio Social) - Departamento de Teoría Sociológica, Filosofía del Derecho y Metodología de las Ciencias Sociales, Universidad de Barcelona, Barcelona, 2001.

ELBOJ, C. et al. Comunidades de aprendizaje. Transformar la educación. Barcelona: GRAÓ, 2002.

EPSTEIN, J.; SHELDON, S. Moving forward: Ideas for research on school, family, and community partnerships. In: CHIFTON, C.; RONALD, S. (eds). Handbook for research in education. California: Sage Publications, 2008, p. 115-150.

FOLIACO, R. G. et al. Índices de riesgo educativo (IREs). Revista Educación y Educadores, Colombia, v. 9, n. 2, p. 11-22, 2006. 
GARCÍA, N. El concepto de familia en la constitución. 2009. Tesis (Licenciatura en Ciencias Jurídicas y Sociales) - Facultad de Derecho, Universidad de Chile, Santiago, 2009.

GERSTENFELD, P. Comparación regional del impacto de las características del hogar en el logro escolar. In: NACIONES UNIDAS. Serie Políticas Sociales. Variables extrapedagógicas y equidad en la educación media: Hogar, subjetividad y cultura escolar. Santiago: CEPAL, 1995, p. 13-37.

HIMMEL, E.; MALTES, S.; MAJLUF, N. Análisis de la influencia de factores alterable del proceso educativo sobre la efectividad escolar. Santiago: Universidad Católica de Chile, 1984.

KERLINGER, E.; LEE, B. Investigación del Comportamiento. Métodos de Investigación en Ciencias Sociales. México: McGraw-Hill, 2002.

LLECE [Laboratorio Latinoamericano de Evaluación de la Calidad de la Educación]; UNESCO [Organización de las Naciones Unidas para la Educación, la Ciencia y la Cultura]. Estudio cualitativo en escuelas latinoamericanas con resultados destacables en siete países latinoamericanos. Santiago: UNESCO, 2002. MAMANI, A.; ARAYA, E. Raíces de nuestro pueblo atacameño, quechua y aymara. Santiago: RIL Editores, 2015. MARRADI, A; ARCHENTI, N; PIOVANI, J. Metodología de las ciencias sociales. 2. ed. Buenos Aires: Cengage Learning, 2012.

MIRANDA, C. El Patrimonio local como herramienta educativa. Revista Internacional de Investigación en Ciencias Sociales. Asunción, v. 13, n. 1, p. 25-34, 2017. https://doi.org/10.18004/riics.2017.julio.25-34

MIRANDA, C.; CASTILLO, P. Estudio metodológico de las variables que componen el constructo de "apoyo y participación de la familia en los procesos educativos". Revista Estudios Pedagógicos, v. 44, n. 1, p. 115133, 2018. https://doi.org/10.4067/S0718-07052018000100115

MOLINA, S. Los grupos interactivos: Una práctica de las comunidades de aprendizaje para la inclusión del alumnado con discapacidad. 2007. 410 f. Tesis (Doctorado de Educación y Democracia) - Departamento de Teoría e Historia de la Educación. Universidad de Barcelona, Barcelona, 2007. Disponible en: http://www. tdx.cat/bitstream/handle/10803/31986/SMR_TESIS.pdf?sequence=1. Acceso en: 30 mar. 2018.

MONTECINO, S. Madres y huachos, alegorías del mestizaje chileno. Santiago: Sudamericana, 1996.

MORALES, H. Etnopolítica atacameña: Ejes de la diversidad. Estudios Atacameños. Arqueología y Antropología Surandinas, n. 53, p. 185-203, 2016. Disponible en: https://scielo.conicyt.cl/pdf/eatacam/n53/ aop1116.pdf. Acceso en: 07 abr. 2018.

MURILLO, F. (coord.). Investigación Iberoamericana sobre Eficacia Escolar. Bogotá: Convenio Andrés Bello, 2007.

NUNNALLY, J. Introducción a la medición psicológica. Buenos Aires: Centro Regional de Ayuda Técnica, 1970.

NUNNALLY, J. Teoría psicométrica. México: Trillas, 1987.

PIZARRO, R. Anexo n. 4 de Proyecto FONDECYT n. 1040251. Santiago: FONDECYT-CONICYT, 2006.

PIZARRO, R. Sistemas educativos formales y efectos Mateo, Regresivo y Robin Hood. Boletín de Investigación Educacional, Santiago, v. 23, n. 2, p. 13-38, 2008. 
PIZARRO, R. Índices de Riesgos Educativos (IREs). Viña del Mar: EV Síntesis y Excelencias Educativas, 2009.

PIZARRO, R. Lenguajes investigativos educacionales: Supuestos, comparaciones, síntesis. Valparaíso: Universidad de Playa Ancha, 2012.

PIZARRO, R. Diseño de Planes para Fortalecer la Formación Inicial Docente en Universidades del Estado. Informes Anexos para uso interno. Informe Documento n. 13. Valparaíso, Chile, 2015.

PIZARRO, R.; CLARK, S. El ambiente educativo del hogar. Diálogos Educacionales, n. 9-10, p. 66-83, 1987.

PIZARRO, R.; CLARK, S. Currículum del hogar y aprendizajes: Interacción versus status. Revista de Psicología de la Universidad de Chile, v. 7, p. 25-34, 1998. Disponible en: http://www.revistapsicologia. uchile.cl/index.php/RDP/article/view/18764. Acceso en: 15 abr. 2018.

PIZARRO, R. et al. Síntesis y evaluación experimental simultáneas de automaticidad en lectura y Currículum del hogar: Dos metodologías potenciadoras del rendimiento académico lector. Proyecto FONDECYT n. 1960137 de 1996. Paper presentado en el Encuentro por la Unidad de los Educadores Latinoamericanos Pedagogía 97, La Habana, Cuba, 1997.

RUÍZ, M. Educación de adultos y familia. In: BERTELY, M. (coord.). Educación, derechos sociales y equidad, Tomo II. México: COMIE-SEP-CESU, 2003, p. 713-743.

UNESCO [Organización de las Naciones Unidas para la Educación, la Ciencia y la Cultura]. Informe de la comisión sobre la educación para el siglo XXI. La Educación Encierra un Tesoro. Paris: UNESCO, 2012.

UNESCO [Organización de las Naciones Unidas para la Educación, la Ciencia y la Cultura]. Eficacia escolar y factores asociados en América Latina y el Caribe. Santiago: UNESCO, 2008.

UNICEF [Fondo de las Naciones Unidas para la Infancia]. Escuelas efectivas en sectores de pobreza, ¿quién dijo que no se puede?, 2004. Disponible en: http://www.unicef.cl/centrodoc/escuelas_efectivas/ escuela\%20efectivas.pdf. Acceso en: 16 abr. 2018.

VALDÉS, C.; CARLOS, E.; ARREOLA, C. Desarrollo de un instrumento para medir la participación de los padres en la educación escolar de los hijos. Revista de Evaluación Educativa, v. 2, n. 1, p. 92-109, 2013. Disponible en: https://www.researchgate.net/publication/285871818_Desarrollo_de_un_instrumento_ para_medir_la_participacion_de_los_padres_en_la_educacion_de_los_hijos. Acceso en: 18 abr. 2018.

VALDÉS, A.; MARTÍN, M.; SÁNCHEZ, P. Participación de padres de alumnos en educación primaria en las actividades académicas de sus hijos. Revista Electrónica de Investigación Educativa, v. 11, n. 1, p. 1-17, 2009. Disponible en: https://redie.uabc.mx/redie/article/view/229/386. Acceso en: 18 mar. 2018.

VALLS, R. Comunidades de aprendizaje: Una práctica educativa de aprendizaje Dialógico para la sociedad de la Información. 2000. 337 f. Tesis (Doctorado Pedagogía Social y Políticas Sociales) - Departamento de Teoría e Historia de la Educación, Universidad de Barcelona, Barcelona, 2000.

VIEIRA, L. Voluntariado en la escuela: Un estudio de casos dentro del proyecto comunidades de aprendizaje. 2010. 461 f. Tesis (Doctorado en Desarrollo Profesional e Institucional para la Calidad Educativa) - Departamento de Didáctica y Organización Educativa, Universidad de Barcelona, Barcelona, 2011. 
YURÉN, M.; CRUZ, M. La relación familia-escuela: Condición de mejora de la eficacia escolar en la formación valoral de niños(as) migrantes. Revista Iberoaméricana sobre Calidad, Eficacia y Cambio en Educación, v. 7, n. 2, p. 131-150, 2009. Disponible en: http://www.redalyc.org/pdf/551/55111725008.pdf. Acceso en: 02 abr. 2018.

ZÁRATE, G. Experiencias educativas exitosas. Un análisis a base de testimonios. Estudios Públicos, n. 47, p. 127-158, 1992.

\section{Sobre los Autores}

Carlos Miranda Carvajal es estudiante del Programa de Doctorado en Psicología, en la línea Psicología y Cambios Sociales en Educación, de la Escuela de Psicología de la Pontificia Universidad Católica de Valparaíso, Chile. Becario de Extensión PUCV-2020. Becario Consejo de Pueblos Atacameños CPA 2020. Magister en Evaluación Educacional y Profesor de Educación Básica con especialización en Educación Rural por la Universidad de Playa Ancha, Chile.

Pablo Castillo Armijo es Profesor Auxiliar del Departamento de Fundamentos de la Educación de la Facultad de Ciencias de la Educación de la Universidad Católica del Maule. Miembro del Centro de Investigación en Educación para la Justicia Social. Doctor en Educación y Sociedad, Master en Investigación en Didáctica, Formación y Evaluación Educativa. Licenciado en Pedagogía por la Universitat de Barcelona, España. Magister en Educación con mención Currículum. Licenciado en Historia por la Pontificia Universidad Católica de Valparaíso, Chile.

Recibido: 13 Ago. 2018

Acepto: 31 Mar 2020 